\title{
DISTRIBUCIÓN ESPACIAL DE LA RUGOSIDAD EN PARCELAS AGRÍCOLAS EN PROVINCIA DE BUENOS AIRES - ARGENTINA
}

\section{HÉCTOR SALGADO}

RESUMO: O uso de imagens SAR para estimar e monitorar a umidade superficial do solo requer que se considere outros fatores que influenciam na retrodifusão do sinal-radar, entre os quais a rugosidade da cobertura da superfície à escala de centímetro é muito importante. Há diversos métodos para determinar a rugosidade, mas muitos são caros ou de operação de campo complexa. Neste trabalho, é apresentado um método versátil e econômico que usa máquina fotográfica e tela quadrada. Cada fotografia é processada numericamente obtendo a altura RMS, como parâmetro da rugosidade da cobertura. Por meio de técnicas geoestatísticas de krigagem é estimada a distribuição espacial da rugosidade. São mostradas experiências em áreas com cobertura de trigo, localizadas na área agrícola serrana da Província o Buenos Aires, Argentina. Os valores de RMS encontrados (29 $\mathrm{mm}<\mathrm{RMS}<48 \mathrm{~mm}$ ) foram analisados com quatro critérios de rugosidade. É expressa sua utilidade para estimar o estado hídrico superficial de solos em áreas agrícolas mediante sua aplicação como entrada (input) nos modelos de retrodispersão de imagens SAR.

PALAVRAS-CHAVE: rugosidade superficial, microtopografia, rugosímetro.

\section{ROUGHNESS SPATIAL DISTRIBUTION IN AGRICULTURAL PARCELS IN BUENOS AIRES PROVINCE, ARGENTINA}

\begin{abstract}
Use of SAR images for soil surface moisture estimation requires taking into account the other factors that influence the radar backscattering signal, among which the surface cover roughness at centimeter scale is very important. There are several methods to determine the roughness, but many are expensive or complex field operation. A versatile and economic method that uses a photographic camera and a girded screen is presented. Each picture is numerically processed obtaining the RMS height, as parameter of the crop-soil complex roughness. By means of krigging geostatistics techniques the spatial distribution of roughness is estimated. Experiences in parcels with wheat cover, located in the hill agricultural area of Buenos Aires Province, Argentina are shown. The found RMS values $(29 \mathrm{~mm}<\mathrm{RMS}<48 \mathrm{~mm})$ are analyzed with four roughness approaches. Their utility in order to estimate soil surface moisture status in agricultural parcels by means of their application like input into the SAR images backscattering models is stated.
\end{abstract}

KEYWORDS: surface roughness, microtopography, roughness meter.

\footnotetext{
${ }^{1}$ Ing. Agrónomo, Agrimensor, Profesor Titular, Facultad de Ciencias Agrarias y Forestales, UNLP, Calles 60 y 119 , La Plata, Argentina, hasalga101@yahoo.com.ar 


\section{INTRODUCCIÓN}

La utilización de imágenes de sensores satelitales de microondas requiere la cuantificación de los elementos de la escena que influyen en la intensidad de la respuesta. Las imágenes provistas por el Radar de Abertura Sintética (SAR) satelital muestran la retrodispersión proveniente de la superficie terrestre del haz de energía emitido por el radar. Diversos factores de la cobertura terrestre influyen en la retrodispersión de la energía, entre ellos, la geometría, su estado de humedad y sus propiedades físico-químicas.

Las estimaciones de la humedad superficial del suelo a partir de imágenes SAR han dado buenos resultados para diversas condiciones de suelo desnudo o con escasa cobertura (WOODING et al., 1992; WEIMANN et al., 1994; DUBOIS et al., 1995; BOISVERT et al., 1996; GENG et al., 1996; ROMBACH AND MAUSER, 1997). Experiencias realizadas en zonas agrícolas de la Provincia de Buenos Aires, Argentina, han arrojado un grado de correlación aceptable, con coeficientes de determinación del orden de 0,67 (SALGADO, 1998; SALGADO et al., 2000, SALGADO et al., 2001). Pero, para ampliar el estudio y aislar la influencia de los demás factores en la retrodispersión, es necesario abordar la medición de la rugosidad del complejo cultivo-suelo en parcelas dedicadas a la agricultura. La rugosidad caracteriza el estado de la superficie de la escena. Entre las propiedades que gobiernan la retrodispersión, una de las más importantes es la microrugosidad, manifestada por las irregularidades de la superficie a escala milimétrica o centimétrica (SABINS, 1986). La rugosidad superficial es una variable relativa, dependiente del sistema de microondas (longitud de onda, polarización, ángulo de incidencia, etc.) que capta la escena (SAHEBI et al., 2002; BAGHDADI et al., 2002 a; SRIVASTAVA et al., 2003). BAGHDADI et al. (2002 b) analizaron también la influencia del ángulo de incidencia en la precisión de estimación de la rugosidad y humedad del suelo, usando redes neuronales.

La presencia de vegetación sobre el suelo constituye un problema adicional para la detección de sus características hidroestructurales por el sistema radar. La retrodispersión de una cobertura vegetal depende de la configuración del sistema radar, de la geometría de la vegetación y de sus propiedades dieléctricas. Así como en el suelo, la constante dieléctrica de la vegetación está también fuertemente ligada a su cantidad en agua (ULABY et al., 1990). La geometría de la vegetación agrupa su macroestructura (altura y densidad de vegetación) y su microestructura (dimensión y distribución de hojas y tallos). La transmisividad de la onda radar en el estrato de vegetación disminuye a medida que la frecuencia, el ángulo de incidencia y la biomasa de la vegetación aumentan (ULABY et al., 1990). El canopeo actúa como atenuador sobre la retrodispersión radar y su efecto es más importante en banda $\mathrm{C}$ que en banda $\mathrm{L}$.

Superficies "suaves o lisas" producen patrones oscuros en las imágenes SAR debido a la reflexión especular del haz de microondas. Por otra parte, superficies altamente rugosas producen dispersión difusa, que se manifiesta con zonas claras-blancas en las imágenes (OH et al., 1992, FUNG, 1994). Situaciones intermedias aparecen en una amplia gama de grises.

La medición de microrugosidad ha concentrado la atención de los investigadores, quienes desarrollaron métodos e ingeniosos instrumentos para cuantificarla. WALL et al. (1991) idearon un sistema de cámaras fotográficas montadas sobre una base-soporte en un helicóptero. Mediante un proceso, similar a una estero-restitución, lograron una representación areal de la rugosidad a escala centimétrica.

Dado que la caracterización de la rugosidad puede hacerse de manera matemática gracias perfiles de la superficie del complejo suelo-vegetación, se han ideado diversos tipos de equipos perfiladores terrestres, desde los más simples y rudimentarios, como el empleo de una regla y la vista humana, hasta los sofisticados perfilómetros Laser. Los sistemas mecánicos, basados en el empleo de rodillos, cadenas o varillas, se "apoyan" sobre la superficie, y por lo tanto no resultan aptos para el canopeo, dado que lo deforman y no reflejan su topografía natural. Los ópticos son mejores, ya que no disturban la superficie de la cubierta vegetal. Entre los ópticos, los perfilómetros Laser son muy precisos, pero caros y difíciles de transportar y operar a campo 
(ROMKENS et al., 1988). También se han empleado cámaras fotográficas, con trípode y flash (PATERSON et al., 1991; BRISCO et al., 1989).

Aquí se presenta un método versátil, económico y fácil de operar, resultante de adaptación del sistema de cámara fotográfica, en el cual se incorporó una pantalla portátil y accesorios de posicionamiento, medición y orientación. El modelo de continuidad espacial se confecciona interpolando mediante un estimador de krigeado ordinario a partir de perfiles captados en las parcelas agrícolas, para obtener el mapa de distribución espacial de la rugosidad.

\section{MATERIAL Y MÉTODO}

Las experiencias se realizaron en parcelas con cultivos de trigo (Triticum spp.) en una importante zona agrícola en el centro de la Provincia de Buenos Aires, Argentina (latitud $37^{\circ} \mathrm{S}$; longitud $\left.60^{\circ} \mathrm{W}\right)$.

El equipo de campaña se compone de una cámara fotográfica, una pantalla y accesorios de medición (cinta métrica, jalones, brújula y posicionador GPS).

Para establecer el grado de rugosidad existen diversos criterios. BEAULIEU et al. (1995) recopilan los citados en Tabla 1. En general los estados de rugosidad de las superficies se determinan por un valor límite del desvío cuadrático medio (RMS) de la altura en función de la longitud de onda $\lambda$ y el ángulo de incidencia del haz radar $\phi$. Sólo en el criterio de ULABY AND DOBSON (1988) el estado de rugosidad no es función del ángulo de incidencia $\phi$.

TABLA 1. Criterios para establecer el grado de rugosidad (BEAULIEU et al., 1995).

\begin{tabular}{|c|c|}
\hline Criterio de Rugosidad & Grado de Rugosidad \\
\hline RAYLEIGH, citado por ELACHI (1988) & $\begin{array}{l}\text { Ligeramente rugosa cuando RMS }<\lambda / 8 \cos \phi \\
\text { Para RMS } \geq \lambda / 8 \cos \phi \text {, la superficie es rugosa }\end{array}$ \\
\hline FRAUNHOFFER, citado por ULABY et al. (1982) & $\begin{array}{l}\text { Lisa cuando RMS }<\lambda / 32 \cos \phi \\
\text { Para RMS } \geq \lambda / 32 \cos \phi \text {, la superficie es rugosa }\end{array}$ \\
\hline PEAKE \& OLIVER (1971) & $\begin{array}{l}\text { Lisa cuando RMS }<\lambda / 25 \cos \phi \\
\text { Para RMS }>\lambda / 4 \text {. cos } \phi \text {, la superficie es rugosa } \\
\text { Intermedia en los demás casos }\end{array}$ \\
\hline ULABY \& DOBSON (1988) & $\begin{array}{l}\text { Lisa cuando RMS }<0,2 \lambda / 2 \pi \\
\text { Para RMS }>\lambda / 2 \pi, \text { la superficie es rugosa }\end{array}$ \\
\hline
\end{tabular}

Por ejemplo, para determinar el grado de rugosidad aplicando el criterio de Fraunhoffer (ULABY et al., 1982), una superficie puede ser considerada electromagnéticamente "suave" si su altura RMS resulta menor que la función siguiente:

altura RMS $<\lambda / 32 \cos \phi$

donde:

altura RMS - desvío cuadrático medio de la altura del canopeo;

$\lambda$ - longitud de onda, $y$

$\phi$ - ángulo de incidencia local.

Para la banda C (de los satélites RADARSAT y ERS), la $\lambda=56,6 \mathrm{~mm}$, para un $\phi=23^{\circ}$, una superficie es suave si su altura RMS es menor que $1,9 \mathrm{~mm}$. Puede deducirse que una superficie "rugosa" para la banda $C$ puede ser suave para la banda $L$ ( $c$ on $\lambda=225 \mathrm{~mm}$ ), dado que ésta última requiere que RMS sea menor que $8 \mathrm{~mm}$ aproximadamente.

Para la orientación azimutal (horizontal) de la cámara fotográfica, se la dispone perpendicular a la traza subsatelital, y en el sentido vertical con un ángulo de incidencia similar al del haz SAR, presentando la pantalla perpendicular (ortogonal) al mismo (Figura 1). 


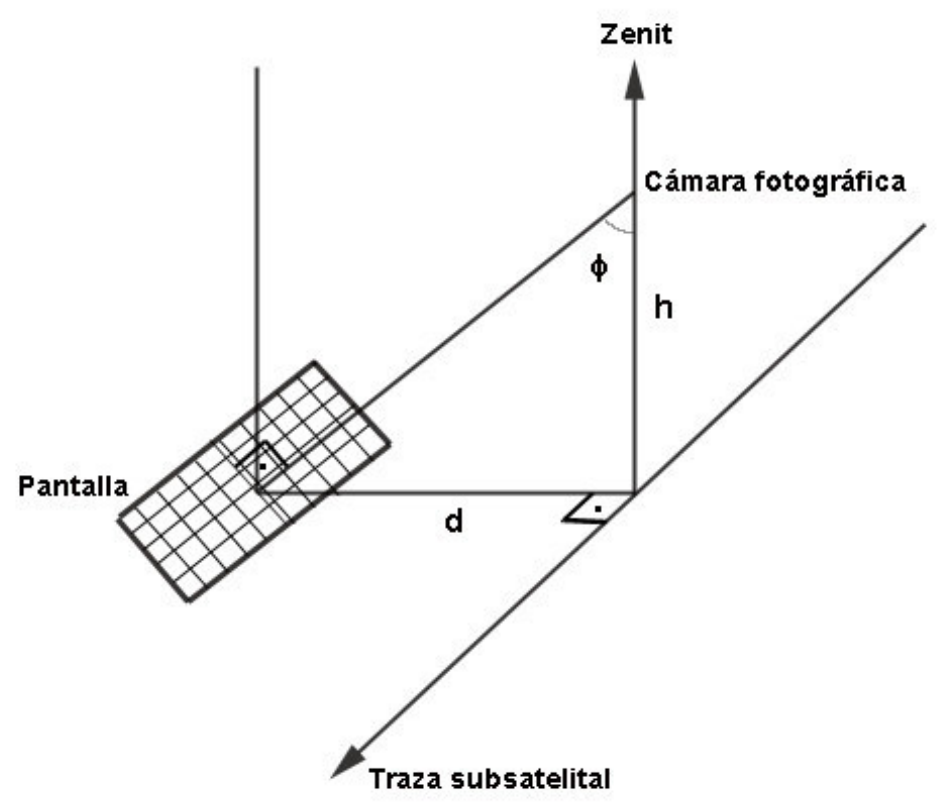

FIGURA 1. Esquema de la configuración de la captura fotográfica, orientada en relación con la traza subsatelital.

La orientación vertical se logra ajustando la distancia "d", se acuerdo a la relación trigonométrica:

$\mathrm{d}=\mathrm{h} \operatorname{tg} \phi$

donde:

h - altura de la toma fotográfica, y

$\phi$ - ángulo de incidencia local.

Por ejemplo, para $\mathrm{h}=1,70 \mathrm{~m}$ y $\phi=40^{\circ}$ resulta $\mathrm{d}=1,43 \mathrm{~m}$. Se emplea el zoom de la cámara para aprovechar el campo focal, de modo que abarque toda la pantalla cuadriculada, obteniendo así la mejor resolución.

Para la orientación horizontal $(\alpha)$ se reprodujo la dirección ortogonal al azimut $(\mathrm{Az})$ de la trayectoria subsatelital. Se utilizó una brújula, con vacilación angular +/- 15', lo cual es suficiente, dada la corta distancia cámara-escena.

$\alpha=\mathrm{Az}+/-90^{\circ}$

La pantalla, de tonalidad clara, se encuentra cuadriculada cada $10 \mathrm{~cm}$, para facilitar la medición de las alturas del canopeo.

Cada fotografía es georreferenciada con un posicionador satelital del tipo receptor GPS GARMIN e-Trex, con un error planimétrico de posición estimado de +/- $5 \mathrm{~m}$.

El proceso requiere la numerización de las fotografías (Figura 2), su escalado, y la colección de alturas $\left(\mathrm{z}_{\mathrm{i}}\right)$ con una distancia de muestreo (e) acorde al sistema SAR con el cual se trabajará. Para el caso de banda $\mathrm{C}$, una $\mathrm{e}=2 \mathrm{~mm}$ resulta suficiente.

Se genera un archivo con las alturas $\left(\mathrm{z}_{\mathrm{i}}\right)$ en función de la distancia $\left(\mathrm{x}_{\mathrm{i}}\right)$, con un par de valores $(\mathrm{x}, \mathrm{z})$ cada intervalo "e". Este archivo es procesado numéricamente, obteniendo la altura RMS para cada fotografía. La altura RMS corresponde al desvío cuadrático medio de las alturas del canopeo, de acuerdo a la expresión:

$$
\text { altura } \mathrm{RMS}=\left(\sum \mathrm{v}_{\mathrm{i}}^{2} / \mathrm{n}-1\right)^{1 / 2}
$$

donde: 
$\mathrm{v}_{\mathrm{i}}$ - son los desvíos entre cada valor de altura observado $\mathrm{z}_{\mathrm{i}}$ y la altura media $\mathrm{Z}$, o sea $\mathrm{v}_{\mathrm{i}}=\mathrm{z}_{\mathrm{i}}-\mathrm{Z}, \mathrm{y}$

$\mathrm{n}$ - número total de alturas registradas (en este caso cada $2 \mathrm{~mm}$, siendo en total 501 registros para cada perfil).

Con los datos de posición geográfica (expresada en coordenadas planas) y RMS se genera un archivo, que es procesado con técnicas geoestadísticas, empleando un estimador de krigeado (OLIVER \& WEBSTER, 1990; GOOVAERTS, 1997). Se obtiene un mapa de distribución espacial de la rugosidad (representada por el parámetro altura RMS) para el tipo de cobertura explorado.

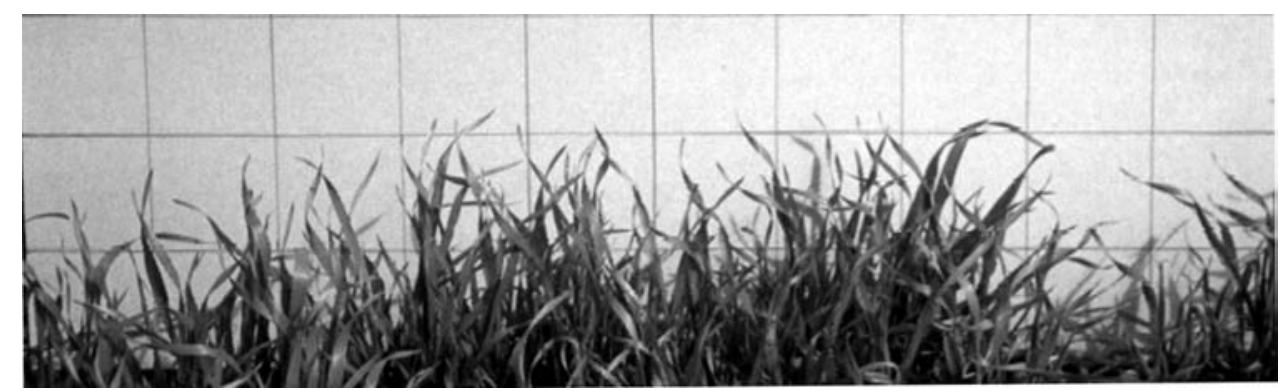

(a)

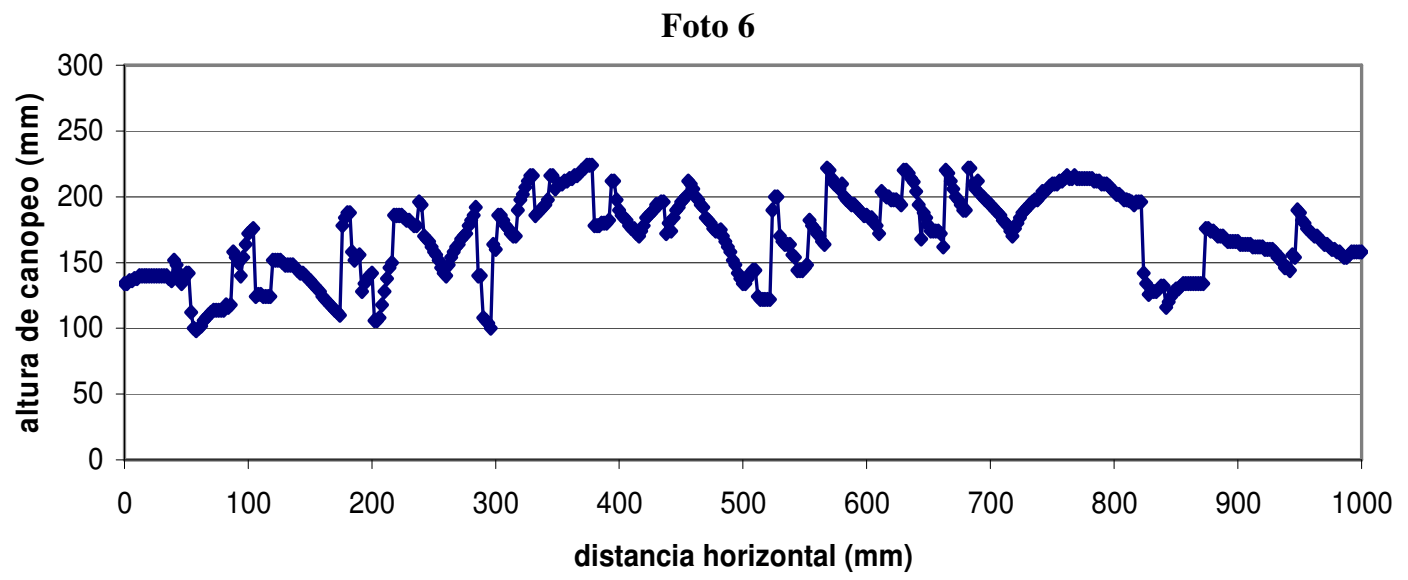

(b)

FIGURA 2. a) Fotografía a campo, con pantalla cuadriculada. (b) Perfil numérico del canopeo.

\section{RESULTADOS}

Los valores de RMS para cada toma fotográfica se presentan en la Tabla 2.

TABLA 2. Valores de rugosidad (RMS) en los 9 sitios medidos.

\begin{tabular}{cccc}
\hline Foto & $\mathrm{X}(\mathrm{m})$ & $\mathrm{Y}(\mathrm{m})$ & RMS $(\mathrm{mm})$ \\
\hline 1 & 5525237 & 5893144 & 28.7 \\
2 & 5525262 & 5893167 & 32.3 \\
3 & 5525280 & 5893187 & 35.9 \\
4 & 5525262 & 5893207 & 48.4 \\
5 & 5525241 & 5893189 & 33.1 \\
6 & 5525219 & 5893166 & 30.8 \\
7 & 5525196 & 5893187 & 29.0 \\
8 & 5525218 & 5893209 & 31.0 \\
9 & 5525239 & 5893233 & 39.7 \\
\hline
\end{tabular}

Los valores extremos se registraron en la foto $1(\mathrm{RMS}=28.7 \mathrm{~mm})$ y en la foto $4(\mathrm{RMS}=$ $48.4 \mathrm{~mm}$ ). Los correspondientes perfiles se muestran en la Figura 3. 
La distribución espacial de la rugosidad se calcula a partir de los valores de RMS anteriormente determinados, mediante una interpolación con un estimador de krigeado ordinario omnidireccional. El mapa obtenido se muestra en Figura 4.

La parcela muestra un aumento gradual de rugosidad hacia la zona norte, donde se encuentra el máximo valor. En este caso la rugosidad va asociada a heterogeneidad en el canopeo, que puede deberse a varios factores del suelo y/o del cultivo.

Es evidente que la rugosidad en coberturas con canopeo denso es altamente variable según las condiciones ambientales. Por ejemplo, la influencia del viento es importante, ya que puede producir un efecto de "peinado o planchado" sobre el cultivo. Por lo tanto, la toma de las fotografías debe hacerse simultáneamente con la captura de la imagen. Esto plantea un problema operativo, que debe ser tenido en cuenta en oportunidad de efectuar estas mediciones en suelos con cobertura vegetal.

Por otra parte, el proceso de orientación de la cámara fotográfica a campo requiere contar con las efemérides satelital, a fines de replantear el azimut de la trayectoria subsatelital.

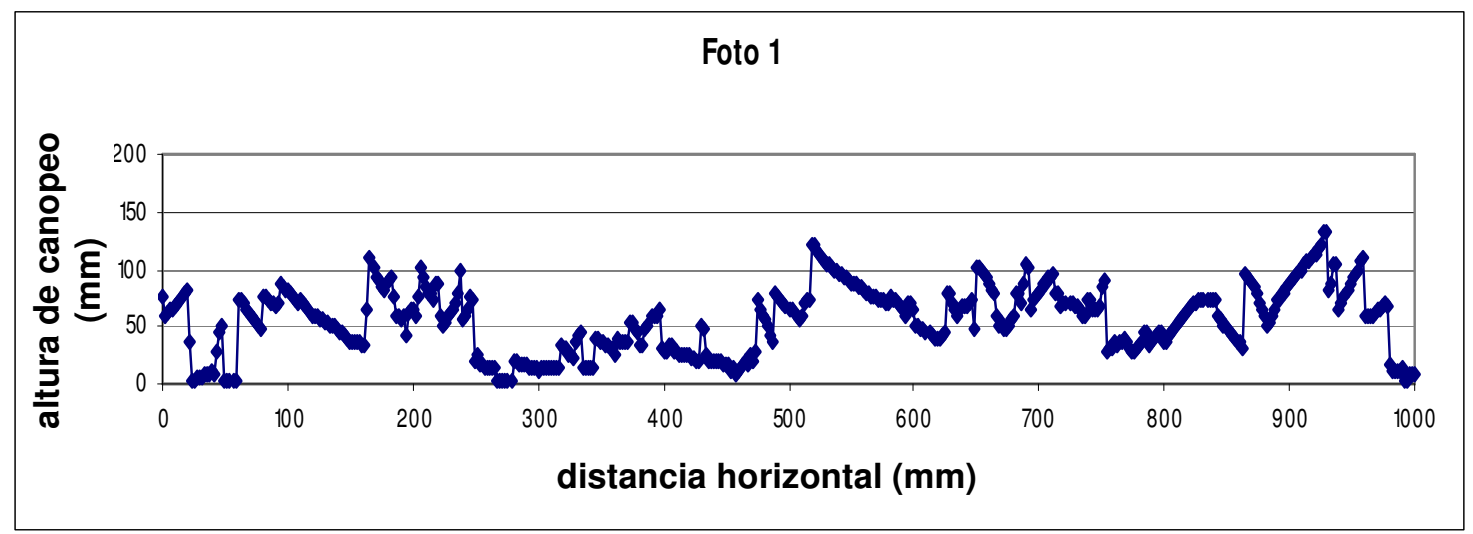

(a)

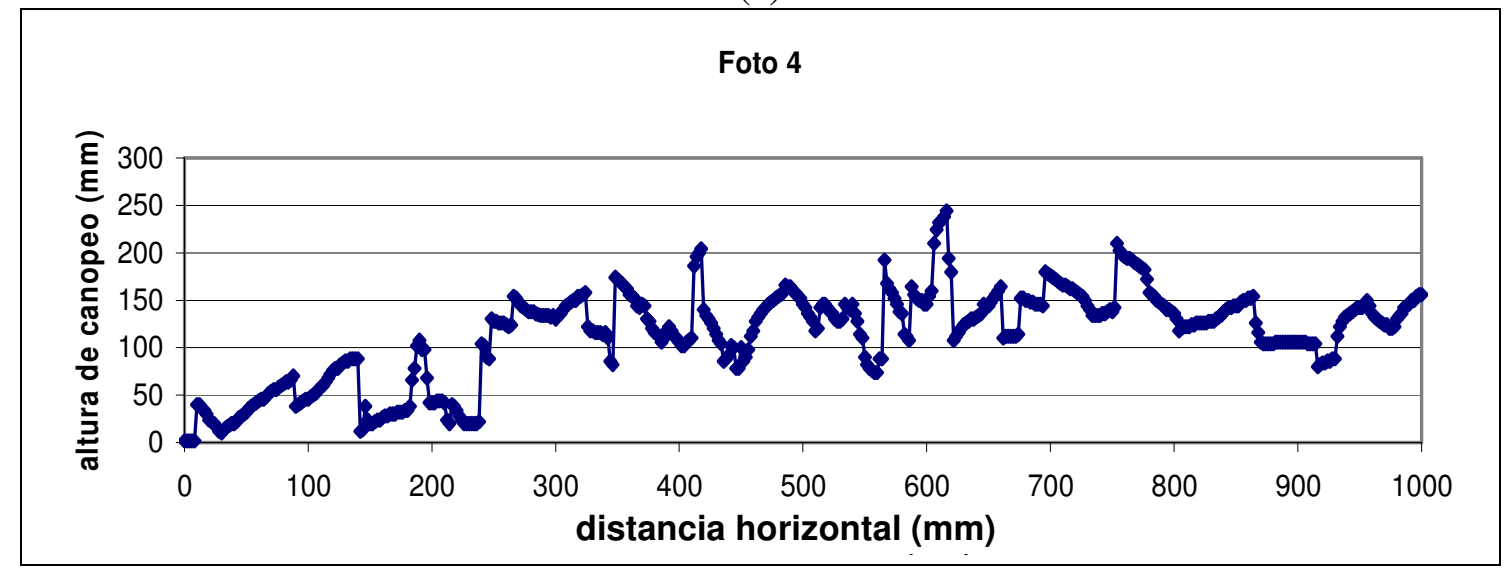

(b)

FIGURA 3: a) Perfil correspondiente a Foto 1 (mínima rugosidad). b) Perfil correspondiente a Foto 4 (máxima rugosidad). 


\section{Curvas de isorugosidad (RMS)}

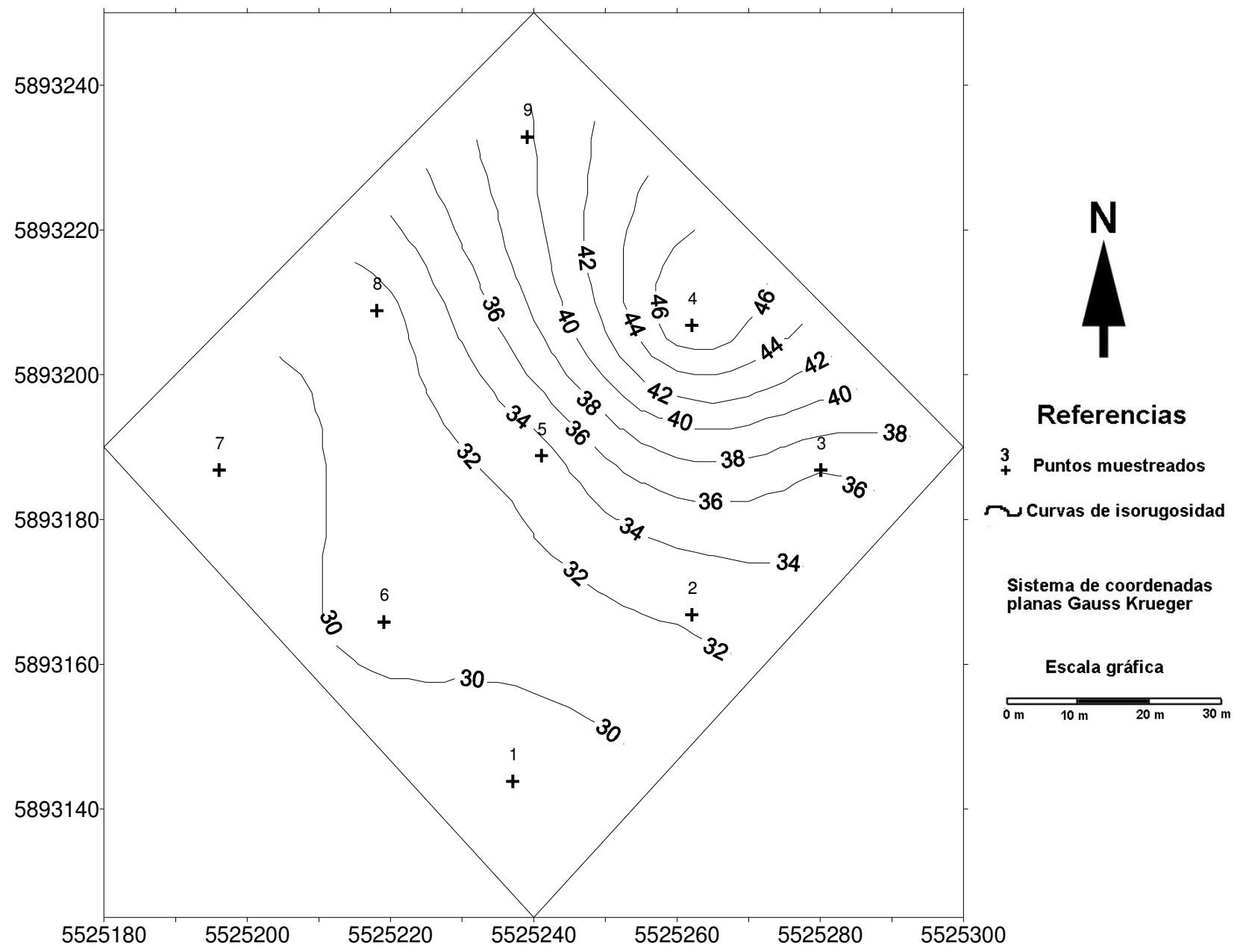

FIGURA 4: Isolíneas RMS en la parcela.

En lo referente a la calificación de rugosidad de superficie, el rango de RMS $(28,7 \mathrm{~mm}<$ RMS $<48,4 \mathrm{~mm}$ ) encontrado para la parcela, se compara con lo valores límite para cada criterio, teniendo en cuenta las características de los 3 sistemas radar más difundidos (bandas X, L y C) y un ángulo de incidencia $\phi=23^{\circ}$, en Tabla 3:

TABLA 3. Aplicación de los criterios de rugosidad.

\begin{tabular}{|c|c|c|c|}
\hline Criterio & Banda $X(\lambda=20 \mathrm{~mm})$ & Banda $\mathrm{C}(\lambda=56 \mathrm{~mm})$ & Banda L $(\lambda=225 \mathrm{~mm})$ \\
\hline Rayleigh & $\begin{array}{c}\mathrm{RMS}>2,7 \mathrm{~mm} \\
\rightarrow \text { rugosa }\end{array}$ & $\begin{array}{c}\text { RMS }>7,6 \mathrm{~mm} \\
\rightarrow \text { rugosa }\end{array}$ & $\begin{array}{c}\mathrm{RMS} \geq 30,5 \mathrm{~mm} \\
\rightarrow \text { rugosa en general }\end{array}$ \\
\hline Fraunhoffer & $\begin{array}{c}\text { RMS }>0,7 \mathrm{~mm} \\
\rightarrow \text { rugosa }\end{array}$ & $\begin{array}{c}\text { RMS }>1,9 \mathrm{~mm} \\
\quad \rightarrow \text { rugosa }\end{array}$ & $\begin{array}{c}\text { RMS }>7,6 \mathrm{~mm} \\
\quad \rightarrow \text { rugosa }\end{array}$ \\
\hline Peake and Oliver & $\begin{array}{c}\mathrm{RMS}>5,4 \mathrm{~mm} \\
\rightarrow \text { muy rugosa }\end{array}$ & $\begin{array}{c}\mathrm{RMS}>15,2 \mathrm{~mm} \\
\rightarrow \text { muy rugosa }\end{array}$ & $\begin{aligned} 9,8 & <\mathrm{RMS}<61,1 \mathrm{~mm} \\
& \rightarrow \text { intermedia }\end{aligned}$ \\
\hline Ulaby and Dobson & $\begin{array}{c}\text { RMS }>3,2 \mathrm{~mm} \\
\rightarrow \text { rugosa }\end{array}$ & $\begin{array}{c}\text { RMS }>8,9 \mathrm{~mm} \\
\rightarrow \text { rugosa }\end{array}$ & $\begin{aligned} 7,2< & <\mathrm{RMS} \leq 35,8 \mathrm{~mm} \\
& \rightarrow \text { intermedia }\end{aligned}$ \\
\hline
\end{tabular}

En general, los valores RMS obtenidos exceden el límite para ser considerada "suave" según los cuatro criterios, por lo cual es esperable que presenten valores significativos de retrodispersión para sensores SAR que operen tanto en banda X como en banda C. Sólo para los criterios de PEAKE AND OLIVER (1971) y de ULABY AND DOBSON (1988), la superficie puede ser admitida como "medianamente rugosa" para imágenes captadas con radar en banda L (como el 
SAR JERS). Esto implica un comportamiento diferencial de las distintas bandas para la misma superficie. En general, cuanto más corta es la longitud de onda, más "sensible" a la rugosidad es el radar.

Unificando los diversos criterios recogidos en la bibliografía, se puede resumir en Tabla 4:

TABLA 4. Clasificación de rugosidad de superficies en función de su RMS y la longitud de onda $\lambda$.

\begin{tabular}{ccc}
\hline Superficie suave o lisa & Superficie con rugosidad intermedia & Superficie rugosa \\
\hline RMS $<\lambda / 25 \cos \phi$ & $\lambda / 25 \cos \phi \leq \mathrm{RMS} \leq \lambda / 4 \cos \phi$ & $\mathrm{RMS}>\lambda / 4 \cos \phi$ \\
\hline
\end{tabular}

\section{CONCLUSIÓN}

El método para representar la distribución especial de la rugosidad puede ser una contribución a la interpretación de la variabilidad espacial de la retrodispersión captada por imágenes SAR en el interior de parcelas agrícolas, y su grado, expresado por RMS, utilizarse como entrada (input) en los modelos de retrodispersión para deducir estado de humedad de los suelos.

\section{REFERENCIAS}

BAGHDADI, N; KING, C.; BOURGUIGNON, A.; REMOND, A. Potential of ERS and Radarsat data for surface roughness monitoring over bare agricultural fields: Application to catchments in Northern France. International Journal of Remote Sensing, United Kingdom, v.23, n.17, p.342742, 2002a.

BAGHDADI, N.; GAULTIER, S.; KING, C. Retrieving surface roughness and soil moisture from synthetic aperture radar (SAR) using neural networks. Canadian Journal of Remote Sensing, Ottawa, v.28, n.5, p.701-11, 2002 b.

BEAUDOIN, A.; GWYN, H.; BORDELEAU, G.; CLICHÉ, P. An economical electronic roughness sampler for radar land studies. In: IGARSS'89, 1989, Vancouver. Proceedings...p.11814.

BEAULIEU, N.; LECLERC, G.; MOISAN, Y. Détermination de la rugosité de surface par des méthodes accessibles. Canadian Journal of Remote Sensing, Ottawa, v.21, n.2, p. 198-203, June 1995.

BERTUZZI, P.; CAUSSIGNAC, J.M.; STENGEL, P.; MOREL, G.; LORENDEAU, J.Y.; PELLOUX, G. An automated, noncontact laser profile meter for measuring soil roughness in situ. Soil Science, Madison, v.149, n.3, p.169-78, March 1990.

BOISVERT, J.B.; PULTZ, T.J.; BROWN, R.J.; BRISCO, B. Potential of synthetic aperture radar for large-scale soil moisture monitoring: A review. Canadian Journal of Remote Sensing, Ottawa, v.22, n.1, p.2-13, March 1996.

BRISCO B.; BROWN, R.J.; CIHLAR, J.; BRANCKER, R.W.S. A field instrument for surface roughness measurement. IGARSS'89, 1989, Vancouver. Proceedings... p.1177-80.

DUBOIS, P.C.; ZIL J. van; ENGMAN, T. Measuring soil moisture with imaging radars. IEEE Transactions on Geoscience and Remote Sensing, New York, v.22, n.4, p.915-26, 1995.

ELACHI, C. Spaceborne radar remote sensing: applications and techniques. New York: IEEE Press, 1988. 255 p.

FUNG, A.K. Microwave scattering and emission models and their applications. Boston: Artech House, 1994. 573 p.

GENG, H.; GWYN, H.; BRISCO,B.; BOISVERT, J.; BROWN, R.J. Mapping of soil moisture from C-Band radar images. Canadian Journal of Remote Sensing, Ottawa, v.22, n.1, p.117-26, March 1996. 
GOOVAERTS, P. Geostatistics for natural resources evaluation. Oxford: University Press, 1997. $482 \mathrm{p}$.

OH Y.; SARABANDI, K.; ULABY, F.T. An empirical model and an inversion technique for radar scattering from bare soil surfaces. I.E.E.E Transactions on Geoscience and Remote Sensing, New York, v.30, n.2, p.370-81, 1992.

OLIVER, M.A.; WEBSTER, R. Kriging: a method of interpolation for Geographical Information Systems. International Journal of Geographical Information Systems, United Kingdom, v.4, n.3, p.313-32, 1990.

PATERSON, J.; BRISCO, B.; ARGUS, S.; JONES, G. In situ measurements of micro-scale surface roughness of sea ice. Journal of the Arctic Institute of North America, Alaska, v.44, Supp.1, p.140-6, 1991.

PEAKE, W.H.; OLIVER, T.L. The response of terrestrial surfaces at microwave frequencies. Columbus: Ohio State University, 1971. (Electroscience Laboratory, 2440-7, Technical Report AFAL-TR-70-301)

ROMBACH, M.; MAUSER, W. Relationships between soil moisture and SAR backscatter. Satellite Data in Hydrology. Experience with ERS., The Netherlands: ESA Publication Division, 1997. p.31-4.

ROMKENS M.J.; WANG, J.Y.; DARDEN, R.W. A laser microreliefmeter. Transactions of the $A S A E$, St. Joseph, v.31, n.2, p.408-13, 1988.

SABINS, F.A. Remote sensing principles and interpretation. $2^{\text {nd }}$ ed. New York: W.H. Freeman, 1986.

SAHEBI M.R.; ANGLES, J.; BONN, F. A comparison of multi-polarization and multi-angular approaches for estimating bare soil surface roughness from spaceborne radar data. Canadian Journal of Remote Sensing, Ottawa, v.28, n.5, p.641-52, 2002.

SALEH, A. Soil roughness measurement: Chain method. Journal of Soil and Water Conservation, United Kingdom, v.48, p.527-9, 1993.

SALGADO, H. Correlación entre humedad del suelo y retrodispersión en imágenes SAR. Revista de la Facultad de Agronomía, Universidad de Buenos Aires, v.18, n.3, p.163-8, 1998.

SALGADO H.; GÉNOVA, L.; ZABALA, S.M.; NIELSEN, Y.J. Soil surface moisture estimation with SAR.PRI images. In: ESA ERS-ENVISAT SYMPOSIUM, 2000, Gothenburg, Sweden, 2000. Proceedings...

SALGADO H.; GÉNOVA, L.; BRISCO, B.; BERNIER, M. Surface soil moisture estimation in Argentina using Radarsat-1 imagery. Canadian Journal of Remote Sensing, Ottawa, v.27, n.6, p.685-90, 2001.

SRIVASTAVA H.; PATEL, P.; MACHANDA, M.L.; ADIGA, S. Use of multiincidence angle of Radarsat-1 SAR data to incorporate the effect of surface roughness in soil moisture estimation. IEEE Transactions on Geoscience and Remote Sensing, New York, v.41, n.7, p.1638-40, 2003.

ULABY, F.T.; MOORE, R.K.; FUNG, A.K. Microwave remote sensing, active and passive. Radar remote sensing and surface scattering theory. Reading, Massachusetts: Addison-Wesley Publishing, 1982. v.2.

ULABY, F.T.; DOBSON, M.C. Handbook of radar scattering statistics for Terrain. Norwwod: Artech House, 1988. 357 p.

ULABY, F.T.; SARABANDI, K.; MCDONALD, K.; WHITT, M.; DOBSON, M.C. Michigan Microwave Canopy Scattering Model (MIMICS). International Journal of Remote Sensing, United Kingdom, v.11, n.7, p.1223-53, 1990. 
WALL S.D.; FAR, T.G.; MULLER, J.P.; LEWIS, P.; LEBERL, F.W. Measurement of surface microtopography. Photogrammetric Engineering \& Remote Sensing, Falls Church, v.57, n.8, p.1075-8, 1991.

WEIMANN A. Evaluation of soil moisture for the Agrometeorological Consulting Service using ERS-1 data (Project PP2-D15). In: ERS-1 PILOT PROJECT WORKSHOP, 1., 1994, Toledo, Spain. Proceedings ... p.93-5.

WOODING, M.; GRIFFITHS, G.H; EVANS, R.; BIRD, P.; KENWARD, D.; KEYTE, G.E. Temporal monitoring of soil moisture using ERS-1 SAR data. In: ERS-1 SYMPOSIUM, 1., 1992, Cannes, France. Proceedings ... p.641-8.

YISOK, O.; KAY, Y.C. Condition for precise measurement of soil surface roughness. IEEE Transactions on Geoscience and Remote Sensing, New York, v.36, n.2, p.691-5, 1998. 